\title{
ASSOCIATIVITY OF THE TENSOR PRODUCT OF SEMILATTICES
}

\author{
by GRANT A. FRASER and JOHN P. ALBERT
}

(Received 19th March 1984)

The tensor product of semilattices has been studied in [2], [3] and [5]. A survey of this work is given in [4]. Although a number of problems were settled completely in these papers, the question of the associativity of the tensor product was only partially answered. In the present paper we give a complete solution to this problem.

For terminology and basic results of lattice theory and universal algebra, consult Birkhoff [1] and Grätzer [6], [7]. The join and meet of elements $a_{1}, \ldots, a_{n}$ of a lattice are denoted by $\sum_{i=1}^{n} a_{i}$ and $\prod_{i=1}^{n} a_{i}$ respectively. All semilattices considered are joinsemilattices. The reader is referred to [2] for definitions and results concerning the tensor product $A \otimes B$ of semilattices $A$ and $B$. In fact, much of [2] is concerned with the special situation in which $A$ and $B$ are distributive lattices, and $A \otimes B$ is obtained by considering $A$ and $B$ as join-semilattices.

We will need the following results from the earlier papers [2, Theorem $2.5 ; 3$, Theorem 1].

Theorem 1. Let $A$ and $B$ be the distributive lattices and let $a, a_{i} \in A$ and $b, b_{i} \in B$ for $i=1, \ldots, n$. Let $n$ be the set $\{1, \ldots, n\}$. Then $a \otimes b \leqq \sum_{i=1}^{n}\left(a_{i} \otimes b_{i}\right)$ in $A \otimes B$ if and only if there exist non-empty subsets $S_{1}, \ldots, S_{m}$ of $n$ such that $a \leqq \sum_{j=1}^{m} \prod_{i \in S_{j}} a_{i}$ and $b \leqq \prod_{i=1}^{m} \sum_{i \in S_{j}} b_{i}$.

Theorem 2. Let $A$ and $B$ be semilattices and let $a, a_{i} \in A$ and $b, b_{i} \in B$ for $i=1, \ldots, n$. Then $a \otimes b \leqq \sum_{i=1}^{n}\left(a_{i} \otimes b_{i}\right)$ in $A \otimes B$ if and only there is an $n$-ary lattice polynomial $p$ such that $a \in p\left(\left(a_{1}\right), \ldots,\left(a_{n}\right)\right)$ and $b \in p^{*}\left(\left(b_{1}\right), \ldots,\left(b_{n}\right)\right)$.

Here $(x)$ denotes the principal ideal generated by $x$ and $p^{*}$ is the polynomial obtained by interchanging the lattice operations in $p$.

Now the partial result on associativity of the tensor product obtained earlier is the following [2, Theorem 5.1].

Theorem 3. Let $A, B$ and $C$ be finite distributive lattices. Then $(A \otimes B) \otimes C$ is isomorphic with $A \otimes(B \otimes C)$.

We shall extend this result first to arbitrary distributive lattices and then to arbitrary semilattices.

Theorem 4. Let $A, B$ and $C$ be distributive lattices. Then $(A \otimes B) \otimes C$ is isomorphic with $A \otimes(B \otimes C)$. 
Proof. Every element of $(A \otimes B) \otimes C$ can be written in the form $\sum_{i=1}^{n}\left[\left(a_{i} \otimes b_{i}\right) \otimes c_{i}\right]$, where $a_{i} \in A, b_{i} \in B$ and $c_{i} \in C$ for $i=1, \ldots, n$. Let $\varphi:(A \otimes B) \otimes C \rightarrow A \otimes(B \otimes C)$ be defined by $\varphi\left(\sum_{i=1}^{n}\left[\left(a_{i} \otimes b_{i}\right) \otimes c_{i}\right]\right)=\sum_{i=1}^{n}\left[a_{i} \otimes\left(b_{i} \otimes c_{i}\right)\right]$. We prove that $\varphi$ is an isomorphism by showing that for all $a_{i}, e_{j} \in A, b_{i}, f_{j} \in B, c_{i}, g_{j} \in C, i=1, \ldots, n, j=1, \ldots, m$, we have $\sum_{i=1}^{n}\left[\left(a_{i} \otimes b_{i}\right) \otimes c_{i}\right] \leqq \sum_{j=1}^{m}\left[\left(e_{j} \otimes f_{j}\right) \otimes g_{j}\right] \quad$ if and only if $\sum_{i=1}^{n}\left[a_{i} \otimes\left(b_{i} \otimes c_{i}\right)\right] \leqq$ $\sum_{j=1}^{m}\left[e_{j} \otimes\left(f_{j} \otimes g_{j}\right)\right]$. Clearly it suffices to prove that $(a \otimes b) \otimes c \leqq \sum_{1}^{n}\left[\left(a_{i} \otimes b_{i}\right) \otimes c_{i}\right]$ if and only if $a \otimes(b \otimes c) \leqq \sum_{1}^{n}\left[a_{i} \otimes\left(b_{i} \otimes c_{i}\right)\right]$. In view of the symmetry of this assertion, it is enough to prove it in one direction.

Suppose that $(a \otimes b) \otimes c \leqq \sum_{1}^{n}\left[\left(a_{i} \otimes b_{i}\right) \otimes c_{i}\right]$. Then by Theorem 1, there are nonempty subsets $S_{1}, \ldots, S_{m}$ of $n$ such that

$$
a \otimes b \leqq \sum_{j=1}^{m} \prod_{i \in S_{j}}\left(a_{i} \otimes b_{i}\right)=\sum_{j=1}^{m}\left(\prod_{i \in S_{j}} a_{i}\right) \otimes\left(\prod_{i \in S_{j}} b_{i}\right)
$$

and

$$
c \leqq \prod_{j=1}^{m} \sum_{i \in S_{j}} c_{i}
$$

Again using Theorem 1, we have that there exist non-empty subsets $T_{1}, \ldots, T_{p}$ of $\boldsymbol{m}$ such that

$$
a \leqq \sum_{k=1}^{p} \prod_{j \in T_{k}}\left(\prod_{i \in S_{j}} a_{i}\right)
$$

and

$$
b \leqq \prod_{k=1}^{p} \sum_{j \in T_{k}}\left(\prod_{i \in S_{j}} b_{i}\right)
$$

Now for $k=1, \ldots, p$, let $U_{k}=\left\{i \in S_{j}: j \in T_{k}\right\}$. Then $U_{1}, \ldots, U_{p}$ are non-empty subsets of $\boldsymbol{n}$. Then by (2) we have

$$
a \leqq \sum_{k=1}^{p} \prod_{i \in U_{k}} a_{i}
$$

Using (1) and (3) we have that for $k=1, \ldots, p, b \leqq \sum_{j \in T_{k}} \prod_{i \in S_{j}} b_{i}$ and $c \leqq \prod_{j \in T_{k}} \sum_{i \in S_{j}} c_{i}$. Then it follows by Theorem 1 that for $k=1, \ldots, p$, we have $b \otimes c \leqq \sum_{i \in U_{k}}\left(b_{i} \otimes c_{i}\right)$. Hence $b \otimes c \leqq \prod_{k=1}^{p} \sum_{i \in U_{k}}\left(b_{i} \otimes c_{i}\right)$. Applying Theorem 1 to the preceding result and (4), we obtain $a \otimes(b \otimes c) \leqq \sum_{1}^{n}\left[a_{i} \otimes\left(b_{i} \otimes c_{i}\right)\right]$.

Theorem 5. Let $A, B$ and $C$ be semilattices. Then $(A \otimes B) \otimes C$ is isomorphic with $A \otimes(B \otimes C)$.

Proof. The initial remarks made in the proof of Theorem 4 remain valid in this case and we define the map $\varphi$ in the same way as before. Again it suffices to prove that if $(a \otimes b) \otimes c \leqq \sum_{i=1}^{n}\left[\left(a_{i} \otimes b_{i}\right) \otimes c_{i}\right]$ then $a \otimes(b \otimes c) \leqq \sum_{i=1}^{n}\left[a_{i} \otimes\left(b_{i} \otimes c_{i}\right)\right]$. Now it follows 
by Theorem 2 that this assertion is equivalent to the statement: if there is an $n$-ary polynomial $p$ such that $a \otimes b \in p\left(\left(a_{1} \otimes b_{1}\right), \ldots,\left(a_{n} \otimes b_{n}\right)\right)$ and $c \in p^{*}\left(\left(c_{1}\right), \ldots,\left(c_{n}\right)\right)$, then there is an $n$-ary polynomial $t$ such that $a \in t\left(\left(a_{1}\right), \ldots,\left(a_{n}\right)\right)$ and $b \otimes c \in t^{*}\left(\left(b_{1} \otimes c_{1}\right), \ldots,\left(b_{n} \otimes c_{n}\right)\right)$.

We will prove this statement by induction on the complexity of the polynomials involved. It is clearly true for polynomials of length 1 . Now assume that the statement holds for the $n$-ary polynomials $p$ and $q$. Let $a \otimes b \in(p+q)\left(\left(a_{1} \otimes b_{1}\right), \ldots,\left(a_{n} \otimes b_{n}\right)\right)$ and $c \in p^{*} q^{*}\left(\left(c_{1}\right), \ldots,\left(c_{n}\right)\right)$. Then $a \otimes b \leqq \sum_{i=1}^{k}\left(x_{i} \otimes y_{i}\right)+\sum_{i=k+1}^{m}\left(x_{i} \otimes y_{i}\right)$ where $x_{i} \in A$ and $y_{i} \in B$ for $i=1, \ldots, m$ and

$$
\sum_{1}^{k}\left(x_{i} \otimes y_{i}\right) \in p\left(\left(a_{1} \otimes b_{1}\right), \ldots,\left(a_{n} \otimes b_{n}\right)\right)
$$

and

$$
\sum_{k+1}^{m}\left(x_{i} \otimes y_{i}\right) \in q\left(\left(a_{1} \otimes b_{1}\right), \ldots,\left(a_{n} \otimes b_{n}\right)\right) .
$$

Thus $a \otimes b \leqq \sum_{1}^{m}\left(x_{i} \otimes y_{i}\right)$, where for each $i$ either $x_{i} \otimes y_{i} \in p\left(\left(a_{1} \otimes b_{1}\right), \ldots,\left(a_{n} \otimes b_{n}\right)\right)$ or $x_{i} \otimes y_{i} \in q\left(\left(a_{1} \otimes b_{1}\right), \ldots,\left(a_{n} \otimes b_{n}\right)\right)$. Since $c \in p^{*}\left(\left(c_{1}\right), \ldots,\left(c_{n}\right)\right)$ and $c \in q^{*}\left(\left(c_{1}\right), \ldots,\left(c_{n}\right)\right)$ it follows that for all $i$ there is an $n$-ary polynomial $s$ (where $s$ is either $p$ or $q$ ) such that

$$
x_{i} \otimes y_{i} \in s\left(\left(a_{1} \otimes b_{1}\right), \ldots,\left(a_{n} \otimes b_{n}\right)\right)
$$

and

$$
c \in s^{*}\left(\left(c_{1}\right), \ldots,\left(c_{n}\right)\right) \text {. }
$$

Now by the inductive hypothesis, for each $i$ there is an $n$-ary polynomial $u_{i}$ such that

$$
x_{i} \in u_{i}\left(\left(a_{1}\right), \ldots,\left(a_{n}\right)\right)
$$

and

$$
y_{i} \otimes c \in u_{i}^{*}\left(\left(b_{1} \otimes c_{1}\right), \ldots,\left(b_{n} \otimes c_{n}\right)\right) .
$$

Since $a \otimes b \leqq \sum_{1}^{m}\left(x_{i} \otimes y_{i}\right)$ it follows by Theorem 2 that there is an $n$-ary polynomial $r$ such that $a \in r\left(\left(x_{1}\right), \ldots,\left(x_{n}\right)\right)$ and $b \in r^{*}\left(\left(y_{1}\right), \ldots,\left(y_{n}\right)\right)$. Let $t$ be the $n$-ary polynomial $r\left(u_{1}, \ldots, u_{n}\right)$. Since $x_{i} \in u_{i}\left(\left(a_{1}\right), \ldots,\left(a_{n}\right)\right)$ for all $i$, it is easy to see that $a \in t\left(\left(a_{1}\right), \ldots,\left(a_{n}\right)\right)$. Also since $b \in r^{*}\left(\left(y_{1}\right), \ldots,\left(y_{n}\right)\right)$ it is readily verified that

$$
b \otimes c \in r^{*}\left(\left(y_{1} \otimes c\right), \ldots,\left(y_{n} \otimes c\right)\right) .
$$

It follows that

$$
\begin{aligned}
b & \otimes c \in r^{*}\left(u_{1}^{*}, \ldots, u_{n}^{*}\right)\left(\left(b_{1} \otimes c_{1}\right), \ldots,\left(b_{n} \otimes c_{n}\right)\right) \\
& =t^{*}\left(\left(b_{1} \otimes c_{1}\right), \ldots,\left(b_{n} \otimes c_{n}\right)\right) .
\end{aligned}
$$

Thus the statement holds for the polynomial $p+q$. 
Finally, assume the statement holds for $p$ and $q$ and suppose that

$$
a \otimes b \in(p q)\left(\left(a_{1} \otimes b_{1}\right), \ldots,\left(a_{n} \otimes b_{n}\right)\right)
$$

and

$$
c \in\left(p^{*}+q^{*}\right)\left(\left(c_{1}\right), \ldots,\left(c_{n}\right)\right) .
$$

Then

$$
\begin{aligned}
& a \otimes b \in p\left(\left(a_{1} \otimes b_{1}\right), \ldots,\left(a_{n} \otimes b_{n}\right)\right), \\
& a \otimes b \in q\left(\left(a_{1} \otimes b_{1}\right), \ldots,\left(a_{n} \otimes b_{n}\right)\right),
\end{aligned}
$$

and there exist $x \in A$ and $y \in B$ such that $c \leqq x+y$ where $x \in p^{*}\left(\left(c_{1}\right), \ldots,\left(c_{n}\right)\right)$ and $y \in q^{*}\left(\left(c_{1}\right), \ldots,\left(c_{n}\right)\right)$. Now by the inductive hypothesis there exist polynomials $r$ and $s$ such that

$$
a \in r\left(\left(a_{1}\right), \ldots,\left(a_{n}\right)\right), b \otimes x \in r^{*}\left(\left(b_{1} \otimes c_{1}\right), \ldots,\left(b_{n} \otimes c_{n}\right)\right),
$$

and

$$
a \in s\left(\left(a_{1}\right), \ldots,\left(a_{n}\right)\right), b \otimes y \in s^{*}\left(\left(b_{1} \otimes c_{1}\right), \ldots,\left(b_{n} \otimes c_{n}\right)\right) .
$$

Let $t$ be the polynomial $r s$. Then $a \in t\left(\left(a_{1}\right), \ldots,\left(a_{n}\right)\right)$ and

$$
b \otimes c \leqq b \otimes(x+y) \in\left(r^{*}+s^{*}\right)\left(\left(b_{1} \otimes c_{1}\right), \ldots,\left(b_{n} \otimes c_{n}\right)\right)
$$

so that

$$
b \otimes c \in t^{*}\left(\left(b_{1} \otimes c_{1}\right), \ldots,\left(b_{n} \otimes c_{n}\right)\right) .
$$

Thus the statement holds for the polynomial $p q$.

This completes the induction and the Theorem is now established.

\section{REFERENCES}

1. G. Birkhoff, Lattice theory, 3rd ed. (Amer. Math. Soc. Colloq. Publ., Vol. 25, Amer. Math. Soc., Providence, R.I., 1967).

2. G. Fraser, The semilattice tensor product of distributive lattices, Trans. Amer. Math. Soc. 217 (1976), 183-194.

3. G. Fraser, The tensor product of semilattices, Algebra Universalis 8 (1978), 1-3.

4. G. Fraser, Tensor products of semilattices and distributive lattices, Semigroup Forum 13 (1976), 178-184.

5. G. Fraser and A. Bell, The word problem in the tensor product of distributive semilattices, Semigroup Forum 30 (1984), 117-120.

6. G. Grätzer, Universal algebra, 2nd ed. (Springer, New York, 1979).

7. G. GRÄTZER, General lattice theory (Academic Press, New York, 1978). 